\title{
Modelling signatures of pulsed magnetopause reconnection in cusp ion dispersion signatures seen at middle altitudes
}

Article

Published Version

Lockwood, M., Davis, C. J., Onsager, T. G. and Scudder, J. D. (1998) Modelling signatures of pulsed magnetopause reconnection in cusp ion dispersion signatures seen at middle altitudes. Geophysical Research Letters, 25 (5). pp. 591-594. ISSN 0094-8276 doi: https://doi.org/10.1029/98GL00185 Available at https://centaur.reading.ac.uk/38758/

It is advisable to refer to the publisher's version if you intend to cite from the work. See Guidance on citing.

Published version at: http://dx.doi.org/10.1029/98GL00185

To link to this article DOI: http://dx.doi.org/10.1029/98GL00185

Publisher: American Geophysical Union

All outputs in CentAUR are protected by Intellectual Property Rights law, including copyright law. Copyright and IPR is retained by the creators or other copyright holders. Terms and conditions for use of this material are defined in the End User Agreement. 


\section{CentAUR}

Central Archive at the University of Reading

Reading's research outputs online 


\title{
Modelling signatures of pulsed magnetopause reconnection in cusp ion dispersion signatures seen at middle altitudes
}

\author{
M. Lockwood, C.J. Davis \\ Rutherford Appleton Laboratory, Chilton, Oxfordshire, UK
}

T.G. Onsager

NOAA/Space Environment Centre, Boulder, USA

and J.D. Scudder

Iowa University, Iowa, USA

\begin{abstract}
It is shown that the open magnetosphere model can reproduce both the down-going and the up-going magnetosheath ions seen in the cusp and mantle regions by the Polar satellite at middle altitudes. The pass studied shows a series of discontinuities in the ion dispersion, most of which are shown to arise from pulses of magnetopause reconnection rate. A total of 9 pulses are detected in an interval estimated to be about 30 min long, giving a mean repetition period of about $3 \mathrm{~min}$ : they vary in length between $0.5 \mathrm{~min}$ and $3.5 \mathrm{~min}$ and are separated by periods of much slower reconnection of duration 1-3 min. One step is not as predicted for reconnection rate pulses but is explained in terms of compressive motions caused by a pulse of solar wind dynamic pressure. The reconnection site is found to be $16 \pm 3 R_{E}$ from the ionosphere along the separatrix field line, placing it at low latitudes on the dayside magnetopause.
\end{abstract}

\section{Introduction}

The cusp ion dispersion signatures caused by pulsed magnetopause reconnection were predicted for low- and midaltitude satellites by Lockwood and Smith [1994]. Lockwood and Davis [1996b] have shown that this theory reproduces observations of cusp ion steps by low-altitude craft when flying over another predicted signature of pulsed reconnection, namely polewardmoving events seen by ground-based instruments. They showed that the signatures detected depend critically on the direction of motion of a low-altitude satellite, relative to the open-closed field line boundary. In the present paper, we show that the theory also predicts the signatures seen by the Polar satellite at middle altitudes. We employ the model by Lockwood [1995] which was designed to reproduce time-varying situations. This open magnetosphere model allows for the evolution of the continuous source population at the magnetopause for each opened field line (as it migrates away from the reconnection site) and for the time-of-flight from the magnetopause to the satellite. The characteristics of this source ion population evolve because of the spatial variation of density and temperature in the magnetosheath and because the rotation angle and speed of the field line at the magnetopause change, altering the acceleration of the ions on crossing the boundary.

Figure 1 shows modelled differential energy fluxes of injected solar wind ions, predicted as a function of ion energy and time

Copyright 1998 by the American Geophysical Union.

Paper number 98GL00185.

0094-8534/98/98GL-00185\$05.00 elapsed since the reconnection of the field line $\left(t_{s}-t_{o}\right.$, where $t_{s}$ is the time that a field line is observed by the satellite and $t_{o}$ is the time when it was reconnected). The inputs required for the model have been listed by Lockwood [1995]: we consider a satellite pass through the cusp at a geocentric distance $r \approx 6 R_{E}$ which is taken to be a field-aligned distance $d_{o}=10.8 R_{E}$ from a subsolar reconnection site. We use the mean solar wind density and temperature measurements from the Wind satellite for the relevant interval of 07:50-08:20 on 20 May $1996\left(N_{s w}=8 \times 10^{6} \mathrm{~m}^{-3}, T_{s w}=1 \times 10^{5} \mathrm{~K}\right)$ with ion transmission and heating factors on crossing the boundary of $t=0.3$ and $h=1.5$, respectively. The magnetosheath field strength at the subsolar point is taken to be $40 \mathrm{nT}$, giving an Alfvén speed of $150 \mathrm{~km} \mathrm{~s}^{-1}$. Figure 1a shows ions at zero pitch angle, i.e. fieldaligned down-going ions. The precipitation evolves with $\left(t_{s}-t_{0}\right)$ in its classification from "(open) LLBL" to "cusp" to "mantle" and "polar cap". Figure 1b shows the solar wind ions with pitch angles at $165^{\circ}$, seen simultaneously moving upward having mirrored below the satellite. Ion flight times are computed using the Tsyganenko T87 model, giving a mirror point about $3.2 R_{E}$ below the satellite. This makes the effective distance from the $\mathrm{X}$-line to the satellite $d_{m} \approx 17.2 R_{E}$ for these up-going ions. The dispersion plume seen in the up-going ions is similar to that in the precipitating ions but expanded along the $\left(t_{s}-t_{o}\right)$ axis because $d_{m}>$ $d_{v}$. Note that after $\left(t_{s}-t_{t}\right)$ of about $400 \mathrm{~s}$, the fluxes of precipitating ions have fallen from "cusp" to "mantle" levels, but considerable fluxes of up-going ions are still seen, as explained using the velocity filter effect by Rosenbauer et al. [1975].

In both cases, a clear minimum energy can be seen at all $\left(t_{s}-t_{0}\right)$, which we here refer to as the low-energy time-of-flight cut-off, $E_{i c}$. For both up- and down-going ions, there is a small spur, before which all the cut-off ions originate from the reconnection site and after which they cross the magnetopause away from the reconnection site at higher magnetic latitudes, as discussed by Lockwood [1995]. To illustrate ion dispersion steps, in the next section we consider the behaviour of $E_{i c}$ in the cusp region.

\section{The effect of reconnection rate variations on ion dispersion}

Lockwood and Smith [1994] and Lockwood and Davis [1996b] considered the form of cusp ion dispersion signatures for various field-perpendicular satellite velocities, relative to the convection velocity: we here consider the components of 


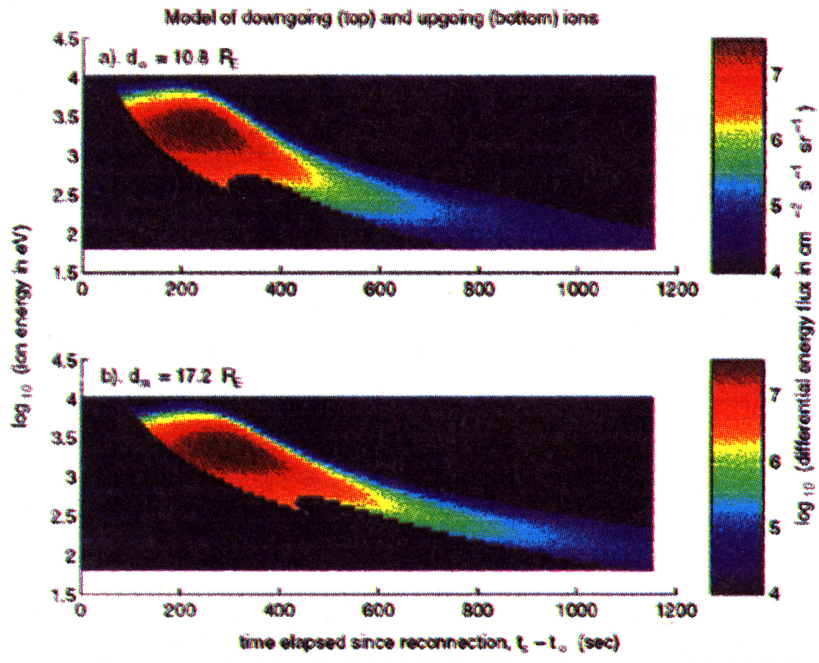

Figure 1. Modelled injected solar wind ions. Differential energy flux of the ions is colour-coded as a function of energy and time elapsed since the field line was reconnected $\left(t_{s}-t_{o}\right)$. The top panel shows ions at zero pitch angle, as observed by a satellite at an altitude which is a field-aligned distance $d_{o}=10.8 \mathrm{R}_{\mathrm{E}}$ from a subsolar reconnection site. The lower panel shows the solar wind ions with pitch angles of $165^{\circ}$, seen simultaneously moving upward having mirrored below the satellite and travelled an effective distance from the $\mathrm{X}$-line to the satellite of $d_{m}=17.2 \mathrm{R}_{\mathrm{E}}$

these velocities that are normal to the open-closed boundary, respectively $V_{s}$ and $V_{c}$ (both defined as positive poleward). For low-altitude satellites moving meridionally $\left|V_{s}\right|>>\left|V_{c}\right|$, whereas for low-altitude satellites moving along the open-closed boundary $\left|V_{s}\right|<\left|V_{c}\right|$. The latter condition will usually also

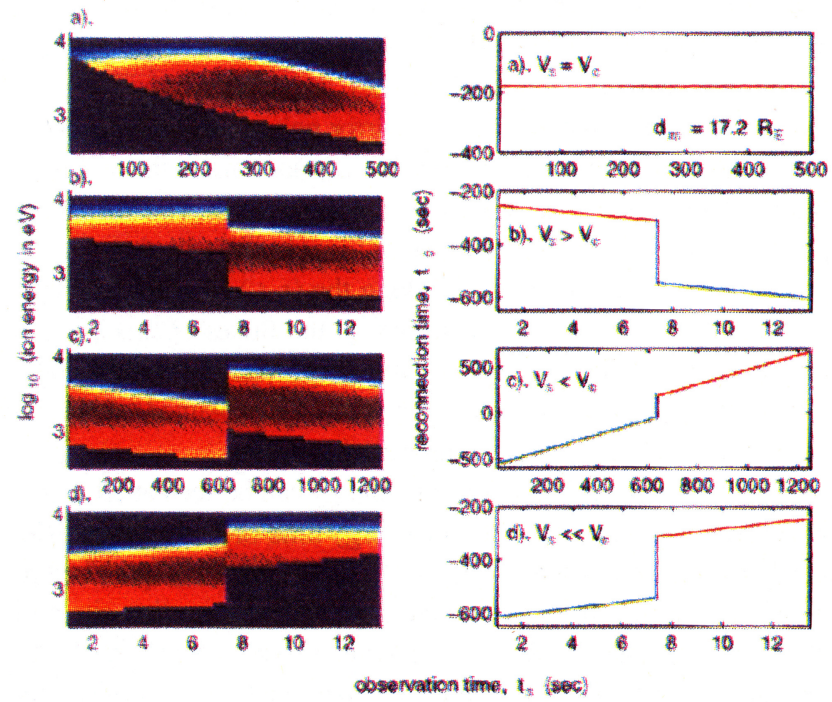

Figure 2. Modelled differential energy flux spectrograms (using the same scale as figure 1), as a function of energy and observation time $t_{s}$ (left); and plots of the field-line reconnection time $t_{o}$, also as a function of $t_{s}$ (right). In all cases, the input reconnection rate $\varepsilon_{y}$ has a square wave variation, falling to zero for an interval of $2.6 \mathrm{~min}$. between two pulses. The green, red and blue segments of the righthand plots arise from the earlier and later pulses and the interval between them, respectively. The satellite is a fieldaligned distance of $d=17.2 \mathrm{R}_{\mathrm{E}}$ from the reconnection site and the conditions are as used in figure 1 . The ratio of the boundary-perpendicular satellite and convection speeds, $V_{s} / V_{c}$ is (a) 1 ; (b) 10 ; (c) 0.1 ; (d) -10 . apply to mid-altitude satellites. Lockwood and Smith (1992) derived an equation from which the magnetopause reconnection rate, $\varepsilon_{y}$, could be computed from observations of $E_{i c}$ in the cusp region. They considered low-altitude satellites and assumed that $V_{c}$ was negligible compared to $V_{s}$ : this assumption was generalised by Lockwood and Davis [1996a] who studied the best implementation and the accuracy of the technique. Inverting their equation (3), we find that the time-of flight low-energy cut-off of the observed ion spectrum, $E_{i c}$, varies with the time of observation, $t_{s}$, with a gradient:

$\mathrm{d} E_{i c} / \mathrm{d} t_{s}=(2 / d)(m / 2)^{-1 / 2} E_{i c}^{3 / 2}\left\{\left[B\left(V_{c}-V_{s}\right)\left(d y / d y^{\prime}\right) / \varepsilon_{y}-1\right\}\right.$

where $d$ is the field-aligned distance from the satellite altitude to the $\mathrm{X}$-line, $m$ is the ion mass, $B$ is the magnetic field at the satellite altitude where a length $d y$ along the open-closed separatrix maps magnetically to a length $d y^{\prime}$ of the X-line.

Equation (1) can be used to illustrate how the dispersion of solar wind ions in the cusp varies with the reconnection rate $\varepsilon_{y}$ by predicting the behaviour of $E_{i c}$. For example, if reconnection is ongoing $\left(\varepsilon_{y}>0\right), \mathrm{d} E_{i c} / \mathrm{d} t_{s}$ is negative if $V_{s}>V_{c}$ (i.e., the ions fall to lower energies with time): on the other hand, if $V_{s}<V_{c}$ the gradient may be positive, negative or even zero. If there is no reconnection taking place $\left(\varepsilon_{y}=0\right), \mathrm{d} E_{i c} / \mathrm{d} t_{s}$ is $-\infty$ (i.e. there is a discontinuous downward step) if $V_{s}>V_{c}$, but is $+\infty$ (a discontinuous upward step) if $V_{s}<V_{c}$. Note that for the special case of $V_{s}=V_{c}$ (i.e. the satellite moves with the flux tube), the dispersion is independent of $\varepsilon_{y}$. This behaviour is reflected in the modelled spectrograms shown in figure 2. The left-hand plots show modelled differential energy flux spectrograms, as a function of energy and observation time $t_{s}$. The plots on the right show the corresponding variation of the field-line reconnection time $t_{o}$, also as a function of $t_{s}$. In all cases, the reconnection rate $\varepsilon_{y}$ has a square-wave variation,

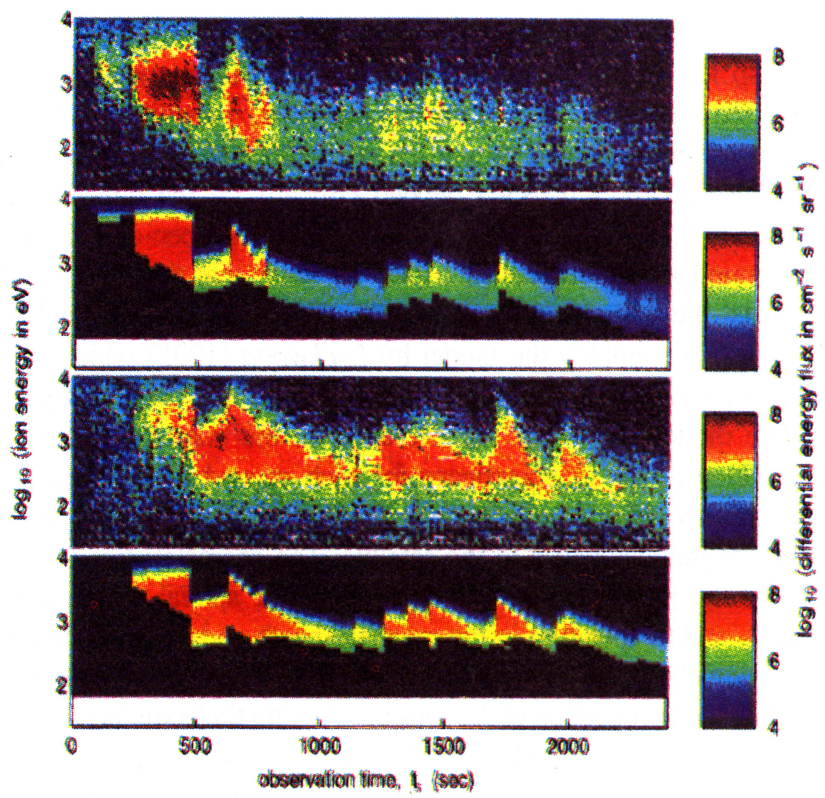

Figure 3. Observed and modelled ion differential energy flux spectrograms, as a function of energy and observation time $t_{s}$, for Polar-Hydra observations on 20 May 1996. Time $t_{s}=0$ is 08:20 UT. From top to bottom: observed down-going ions of pitch angle $0-20^{\circ}$; modelled down-going ions; observed upgoing ions of pitch angle $160-180^{\circ}$; modelled up-going ions. 


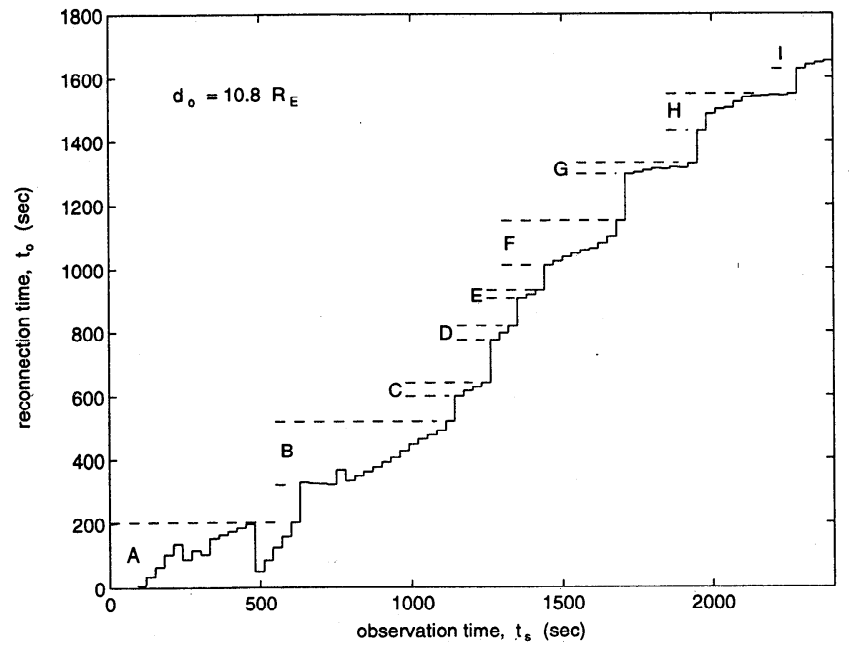

Figure 4. The variation of field-line reconnection time $t_{o}$, as a function of its observation time $t_{s}$, used to generate the fits shown in figure 3 . Pulses of non-zero reconnection rate are delineated by dashed lines and labelled A-I. The plot uses the field-aligned distance from the satellite to the reconnection site of $d_{o}=10.8 \mathrm{R}_{\mathrm{E}}$.

falling to zero for an interval of $2.6 \mathrm{~min}$. between two pulses. Each pulse of reconnection generates a patch of newly-opened flux. The satellite is a field-line distance of $d=17.2 R_{E}$ from the reconnection site and the other conditions and colourbar are as used in figure 1 . In figure 2(a) $V_{s}=V_{c}$, so the satellite monitors the same newly-opened field line and never intersects a step between the two patches. In 2(b), $V_{s}=10 V_{c}$ and the satellite sees, as predicted from (1), downward dispersion ramps within each patch and a downward step as it flies from the more recently-opened patch (shown by the red segment of the right-hand plot) into a patch that was opened earlier (the green segment). In 2(c), $V_{s}=0.1 V_{c}$ and the satellite still sees downward dispersion ramps within each patch of newly-opened flux but an upward step as the patch of more recently-opened flux (again shown in red) is convected over it. Lastly, 2(d) is for $V_{s}=-10 V_{c}$ and the satellite sees upward dispersion ramps within each patch newly-opened flux and an upward step as it flies rapidly equatorward from the older patch (green) into the more recently-opened patch (red): thus 2 (d) is the converse of 2(b). Note that the plots shown on the right hand side of figure 2 are important confirmation that the step is caused by a reconnection pulse as the vertical blue segments correspond to the $2.6 \mathrm{~min}$ interval of zero reconnection rate between the two pulses.

\section{Modelling observations by the Polar satellite}

The top and third panels of figure 3 show, respectively, the down-going and the up-going ions seen by the Hydra ion detector [Scudder et al., 1995] during a pass of the Polar satellite though the cusp region on 20 May 1996. This pass and the prevailing conditions will be described by Onsager et al. [1998]. In the present paper, we are concerned with the fine structure seen in the cusp and mantle regions. The figure shows $40 \mathrm{~min}$. of data starting at $t_{s}=0$, which is 08:20 UT. In this interval, the (MLT, $\Lambda, r$ ) co-ordinates of Polar change from $\left(11: 30,78.2^{\circ}, 6.0 R_{E}\right)$ to $\left(12: 21,81.7^{\circ}, 6.6 R_{E}\right)$. The down-going ions are integrated over a pitch angle range of 0 - $20^{\circ}$ whereas the up-going ions are at $160-180^{\circ}$. Energy-time pixels not sampled in these pitch angle ranges have not been interpolated and are shown as white. It can be seen that the data show steps in both cusp and mantle ions of the kinds predicted in the previous section. In particular, at $t_{s}$ near $500 \mathrm{~s}$ the ions show a downward step like that predicted in figure 2(b), whereas after $t_{s}$ of about $600 \mathrm{~s}$, a series of upward steps like that predicted in figure 2 (c) are observed, particularly in the up-going ions. In this case, Polar has $V_{s}$ of only $0.8 \mathrm{~km} \mathrm{~s}^{-1}$ (for an open/closed boundary that is L-shell aligned) and we would expect $V_{s}<V_{c}$ for antisunward convection. Hence, although the upward steps are expected (figure $2 \mathrm{c}$ ), the downward step is surprising as it requires $V_{s}>V_{c}$ (figure 2b).

The second and fourth panels of figure 3 show fits to these data, made using the variations of the modelled ion spectra with $\left(t_{s}-t_{o}\right)$ shown in figure 1 . The procedure adopted was to generate a fit to the observed up-going ions by selecting a best-fit value of $\left(t_{s}-t_{o}\right)$ for each $t_{s}$. Note that, as demonstrated by the right-hand plots of figure 2 , the $\Delta\left(t_{s}-t_{o}\right) / \Delta t_{s}$ employed specifies the ratio $V_{s} / V_{c}$. The down-going ions then provide an independent test of this selection. It can be seen that agreement to both sets of data is very good; however, it should be noted that several inputs to the model are not known and typical values have been adopted instead: values used have been derived from other measurements, see Lockwood [1995; 1997]. From the best-fit value of $\left(t_{s}-t_{o}\right)$ at each $t_{s}$, the variation of $t_{o}$ with $t_{s}$ shown in figure 4 is obtained.

After $t_{s}=500 \mathrm{~s}$, a number of steps in $t_{o}$ are seen in figure 4, as in the right-hand part of figure $2(\mathrm{c})$. Specifically, periods when reconnection is ongoing (delineated by dashed lines and marked B-I) are seen separated by vertical steps, corresponding to periods when the reconnection rate is much lower. However, figure 4 shows that the step before $t_{s}=500 \mathrm{~s}$ is not between patches of field lines reconnected at different times. This result is found to hold, irrespective of the value of $d_{o}$ adopted. Rather, all field lines seen prior to $t_{s}=500 \mathrm{~s}$ were reconnected at some time within one burst, labelled $A$.

The reconnection pulses revealed by figure 4 vary in length between $0.5 \mathrm{~min}$ and $3.5 \mathrm{~min}$, whereas the periods of low (possibly zero) rate have durations in the range 1.2-2.9 $\mathrm{min}$. In all, 9 pulses of reconnection are seen in the 30 min interval of inferred $t_{o}$, giving a mean repetition period of $3.3 \mathrm{~min}$. Note that the ratio $d_{m} / d_{o}$ cannot be altered by more than about $20 \%$ before the agreement on down-going ions shown in figure 3 begins to degrade for a best fit to the up-going ions: given that the value of $\left(d_{m}-d_{o}\right)$ is set by the altitude of the satellite, the uncertainty in the inferred value of $d_{o}=10.8 R_{E}$ can be estimated to be about $\pm 3 R_{E}$. Thus the reconnection site is inferred to be at a distance of $15.8 \pm 3 R_{E}$ from the ionosphere,-i.e. near the low-latitude magnetopause. This matches a similar the result obtained by Phillips et al. [1993]. This uncertainty in $d_{o}$ is not enough to alter the general form of figure 4 .

\section{Discussion and Conclusions}

A key point about the model used to fit both the up-going and the down-going ions in figure 3 is that it is an open magnetosphere model, in which plasma streams continuously across the magnetopause along each newly-opened field line. As a result, the extent of the magnetopause source region supplying magnetosheath plasma to any one satellite location, is large (10-20 $R_{E}$ ), as recently demonstrated by Lockwood [1997]. The data clearly remove any possibility of the steps in 
the ion dispersion being impulsive injections of plasma over the magnetopause (sometimes termed "plasma transfer events") [Woch and Lundin, 1992]. There are two reasons for this. Firstly, any change in the rate of plasma transfer over the boundary would be seen first in higher energy ions which have a lower flight time: neither the top nor the third panel show any such dispersion at the steps. Furthermore, the same features can be recognised in the up-going and the downgoing ions at the same time, without any delay. Because of the time taken for the observed up-going ions to fly from the satellite, down to the mirror point and back up to the satellite (i.e. over a distance of about $2 \times 3.2 \mathrm{R}_{\mathrm{E}}$ ), this delay would be respectively $29 \mathrm{~s}, 93 \mathrm{~s}$ and $290 \mathrm{~s}$ for protons of eriergy $10 \mathrm{keV}$, $1 \mathrm{keV}$ and $100 \mathrm{eV}$. Any change in the down-going ions caused by a modulation of the flux injected would be seen in the up-going ions only after these delays. On the other hand, the instantaneous steps at all energies and in both up- and downgoing ions is consistent with the concept of continuous (but evolving) injection onto each field line, which is predicted by the open magnetosphere model, but with discontinuous changes in the time when field lines are opened, i.e. pulsed reconnection. Upwarc/downward steps are caused by moving to field lines which were reconnected more/less recently.

As discussed in the previous section, the upward steps in the dispersion ramp after $t_{s}=500 \mathrm{~s}$ are consistent with reconnection rate pulses separated by short (1-3 min) intervals of much slower reconnection. The interplanetary medium during this interval was monitored by the Wind spacecraft [see Onsager et al., 1998] and showed that the IMF was predominantly southward, but with three brief swings to a northward orientation (1-2 min long) around 7:45 UT and two more around 8:05. Neither the propagation delay from Wind to the magnetopause nor the distance $d_{o}$ are known with sufficient accuracy to associate the IMF variations with the inferred reconnection rate variations. For the observed solar wind speed, a nominal lag of $30 \mathrm{~min}$. is estimated, which would place any effects of the brief northward swings around $t_{o}$ of $-300 \mathrm{~s}$ and $900 \mathrm{~s}$. Thus, it is entirely possible that one or two of the periods of slow reconnection follow these brief periods of northward IMF with the appropriate delay; however, there is no lag that makes it possible that they all do. Thus we can infer that although one or two of the reconnection pulses may have been driven by IMF orientation changes, it is not possible that they all were.

The downward step seen near $t_{s}=500 \mathrm{~s}$ is not consistent with reconnection rate pulses because the reconnection times $t_{o}$ show the field lines seen before and after the step were opened in different parts of the same interval A in figure 4. A further indication that there is an additional factor present is that the dispersion ramp seen at $t_{s}=500-600 \mathrm{~s}$ has the opposite sense to that expected, showing that the satellite is moving onto more-recently opened field lines (whilst remaining in the patch produced by pulse $\mathrm{A}$ ), despite the fact that it is moving (slowly) poleward. Lockwood and Smith [1994] noted a key point about mid-altitude observations was that, unlike in the ionospheric case, the magnetic field was compressible and so the field lines could readily be moved back and forth over the satellite by compressions and rarefactions of the dayside magnetosphere by changes in the dynamic pressure of the solar wind flow. The observations by Wind show a rise in the solar wind speed at 7:38-7:46, followed by a short pulse in the density which peaks at 7:50 at roughly twice its average level. With a lag of $30 \mathrm{~min}$., these changes would influence the
Polar observations at around $t_{s}=300 \mathrm{~s}$. The most likely explanation is that a travelling convection twin-vortex (TCV), driven by this pressure pulse, passed over Polar giving a tripolar variation in the velocity $V_{c}$ as, for example, observed by Lühr et al. [1995]. If during the passage of the TCV, $V_{c}$ turned strongly negative (sunward flow), the older open field lines (larger $t_{s}-t_{o}$ ) could be brought rapidly back over the satellite, allowing the downward step seen at $t_{s}=500 \mathrm{~s}$. Subsequently, $V_{c}$ would turn strongly positive and these field lines would be moved back over the satellite, giving the reverse dispersion seen at $t_{s}=500-600 \mathrm{~s}$. This would also hasten the upward step at $t_{s}=600 \mathrm{~s}$ as flux opened by the subsequent reconnection pulse (B) is brought over the satellite for the first time.

Thus the observed ion dispersion is consistent with a mixture of the effects of pulsed reconnection, complicated by the passage of a TCV along the polar cap boundary. We conclude that step features in the mid-altitude ion spectrogram can be produced by compressive motions of the dayside magnetospheric plasma, as well as by reconnection pulses. Because of this complication, an analysis of the reconnection time, $t_{o}$, is required if pulsed reconnection is to be identified. This is presented here in figure 4 and shows the reconnection to be taking place almost exclusively in a series of pulses, separated by brief periods of much slower reconnection.

Acknowledgements. ML and CJD are supported by the UK Particle Physics and Astronomy Research Council.

\section{References}

Lockwood, M., The location and characteristics of the reconnection X-line deduced from low-altitude satellite and ground-based observations: 1 . Theory, J. Geophys. Res., 100, 21791-21802, 1995.

Lockwood, M, Energy and pitch angle dispersions of LLBL/cusp ions seen at middle altitudes: predictions by the open magnetosphere model, Annales Geophys., 15, 1501-1514, 1997.

Lockwood, M., and C.J. Davis, An analysis of the accuracy of magnetopause reconnection rate variations deduced from cusp ion dispersion characteristics, Annales Geophys., 14, 149-161, 1996a.

Lockwood, M., and C.J. Davis, On the longitudinal extent of magnetopause reconnection bursts, Annales Geophys., 14, 865-878, 1996b.

Lockwood, M., and M.F. Smith, Low- and mid-altitude cusp particle signatures for general magnetopause reconnection rate variations: I Theory, J. Geophys. Res., 99, 8531-8555, 1994.

Lühr, H., et al., Multi-instrument ground-based observations of a Travelling Convection Vortex event, Annales Geophys., 14, 162-181, 1996.

Onsager, T.G., et al., Geophys. Res. Lett., to be submitted, 1998.

Phillips, J.L., et al. Well-resolved observations by ISEE 2 of ion dispersion in the magnetospheric cusp, J. Geophys. Res., 98, 13429-13440, 1993.

Rosenbauer, H., et al., HEOS plasma observations in the distant polar magnetosphere: the plasma mantle, J. Geophys. Res., 80, 223-2737, 1975.

Scudder et al., Hydra - a 3-dimensional electron and ion hot plasma instrument for the Polar spacecraft of the GGS mission, Space Sci. Rev., 71, 459-496, 1995.

Woch, J. and R. Lundin, Signatures of transient boundary layer processes observed with Viking, J. Geophys. Res., 97, 1431-1447, 1992.

C.J. Davis and M. Lockwood, RAL, Chilton, Didcot, Oxfordshire, OX11 0QX, England, UK. (e-mail: m.lockwood@rl.ac.uk)

T.G. Onsager, NOAA/Space Environment Center, R/E/SE, $325 \mathrm{Br}-$ oadway, Boulder, CO80303, USA (e-mail: tonsager@ sec.noaa.gov)

J.D. Scudder, The University of Iowa, Department of Physics \& Astronomy, 506 Van Allen Hall, Iowa City, IA 52242-1479, USA (email: jds@space-theory.physics.uiowa.edu)

(Received: November 6, 1997 ; revised: December 18, 1997; accepted: December 18, 1997.) 